\title{
Assessment of the Inhibitory Effect of Rifampicin on Amyloid Formation of Hen Egg White Lysozyme: Thioflavin T Fluorescence Assay versus FTIR Difference Spectroscopy
}

\author{
Gang Ma, Hong Zhang, Jianhua Guo, Xiaodan Zeng, Xiaoqian Hu, and Wenying Hao \\ Key Laboratory of Medicinal Chemistry and Molecular Diagnosis of Ministry of Education, \\ College of Chemistry and Environmental Science, Hebei University, Baoding 071002, China \\ Correspondence should be addressed to Gang Ma; gma.hbu@gmail.com
}

Received 6 September 2013; Revised 31 October 2013; Accepted 14 December 2013; Published 6 January 2014

Academic Editor: Yizhuang Xu

Copyright (C) 2014 Gang Ma et al. This is an open access article distributed under the Creative Commons Attribution License, which permits unrestricted use, distribution, and reproduction in any medium, provided the original work is properly cited.

\begin{abstract}
The inhibitory effect of rifampicin on the amyloid formation of hen egg white lysozyme was assessed with both Thioflavin T (ThT) fluorescence assay and Fourier transform infrared (FTIR) difference spectroscopy. We reveal that ThT fluorescence assay gives a false positive result due to rifampicin interference, while FTIR difference spectroscopy provides a reliable assessment. With FTIR, we show that rifampicin only has marginally inhibitory effect. We then propose that FTIR difference spectroscopy can potentially be a convenient method for inhibitor screening in amyloid study.
\end{abstract}

\section{Introduction}

Amyloid aggregates are insoluble protein aggregates with the characteristic cross- $\beta$ structure and fibrous morphology [1]. In vivo, amyloid deposition is a pathological hallmark of more than 40 neurodegenerative, systemic, and nonsystemic diseases [2]. These human diseases, collectively termed as amyloid diseases, include some well-known disorders such as Alzheimer's disease, Parkinson's disease, and Type II diabetes. Each of these diseases is associated with amyloid fibrillation of one unique type of protein or peptide [2]. The apparent linkage between amyloid formation and amyloid disease makes designing and developing molecular interventions into amyloid formation an attractive strategy to prevent and treat amyloid diseases [3].

Amyloid formation is a rather complex protein aggregation process. The aggregation kinetic curve usually follows a typical sigmoidal function featuring a lag phase, a growth phase, and an equilibrium phase [4]. Furthermore, there can be oligomeric intermediates in diverse sizes and morphologies. These intermediate oligomers have been referred to in numerous ways as amorphous aggregates, protofibrils, prefibrillar aggregates, globulomers, and annular protofibrils [5]. Both mature fibril and nonfibrillar oligomers have been hypothesized to be the possible pathogenic agents in amyloid diseases [3].

As amyloid fibril has been hypothesized to be one of the pathogenic agents in amyloid diseases [3], searching molecules that can inhibit amyloid fibrillation has been one of the major research efforts in the field of amyloid research. Rifampicin is an antibiotic which has been found to be able to inhibit amyloid formation of numerous proteins such as amyloid- $\beta, \alpha$-synuclein, islet amyloid polypeptide (IAPP), and hen egg white lysozyme (HEWL) [6-9]. However, the general picture of the inhibitory effect of rifampicin on amyloid formation has been challenged in recent years. Meng et al. reported that rifampicin in fact had no inhibitory effect on the amyloid formation of IAPP, contrary to previous observation [10]. They demonstrated that the apparent inhibitory effect was simply due to the false positive result of Thioflavin $\mathrm{T}(\mathrm{ThT})$ fluorescence assay caused by rifampicin interference. The discovery by Meng et al. clearly demonstrates that the effect of rifampicin on the amyloid formation is a rather complicated issue and there is no general answer with respect to the actual role of rifampicin during amyloid formation. In this study, we would like to reexamine the inhibitory effect of rifampicin on the amyloid fibrillation of HEWL by using HEWL as a model amyloidogenic system. HEWL is not 
a protein which is directly relevant to amyloid disease, yet it is a widely used model protein in amyloid study due to its low cost. Our aim in this study is threefold. (1) Can rifampicin really inhibit the amyloid formation of HEWL? (2) Does rifampicin interfere with ThT assay in the HEWL system? (3) If the ThT assay is problematic in inhibitor screening, can we find another convenient way to accurately assess the effect of rifampicin?

\section{Material and Methods}

2.1. Materials. Hen egg white lysozyme (HEWL) with a purity of $\geq 90 \%$ (catalog number L6876) was obtained from Sigma-Aldrich (Saint Louis, USA). Deuterium oxide $\left(\mathrm{D}_{2} \mathrm{O}\right)$ with $>99.8 \%$ purity was obtained from J\&K Chemical (Beijing, China). Sodium chloride with $>99 \%$ purity was obtained from Sigma-Aldrich. Deuterium chloride $(\mathrm{DCl})(35 \%$ by weight in $\mathrm{D}_{2} \mathrm{O}$ ) was obtained from Sigma-Aldrich. Sodium azide $\left(\mathrm{NaN}_{3}\right)$ with a purity of $99 \%$ was purchased from Tianjin Chemical Plant (Tianjin, China). Rifampicin was obtained from Yuanye (Shanghai, China). All materials are used without further purification.

2.2. Sample Preparations. HEWL solution in $\mathrm{D}_{2} \mathrm{O}(\mathrm{pD}=2)$ containing $140 \mathrm{mM} \mathrm{NaCl}$ and $200 \mathrm{ppm} \mathrm{NaN}_{3}$ was used for fibrillation study. Solution $\mathrm{pH}$ was adjusted using $\mathrm{DCl}$ solution in $\mathrm{D}_{2} \mathrm{O}$ to reach a $\mathrm{pH}$ meter reading of $1.6(\mathrm{pD}=\mathrm{pH}$ reading +0.4 ). The HEWL solution was first passed through a $0.22 \mu \mathrm{m}$ filter before incubation. Rifampicin was first dissolved into $\mathrm{D}_{2} \mathrm{O}(\mathrm{pD}=2)$ to make a concentrated solution of $7 \mathrm{mM}$. This solution was then used to make HEWL-rifampicin mixture solution. In this study, the concentration of HEWL was $5 \mathrm{mg} / \mathrm{mL}$ and the concentration of rifampicin was $400 \mu \mathrm{M}$. The concentration of HEWL was determined by weighing and the concentration of rifampicin was determined with UV-Vis spectroscopy with the reported extinction coefficient of $28000 \mathrm{M}^{-1} \cdot \mathrm{cm}^{-1}$ at $334 \mathrm{~nm}$ [11]. The HEWL and HEWLrifampicin solutions in sealed glass vials were incubated in a thermoshaker at $62^{\circ} \mathrm{C}$ without agitation. Whenever necessary, aliquots will be taken out of the incubation vial for ThT fluorescence, Fourier transform infrared (FTIR), and atomic force microscopy (AFM) characterizations. Care was always taken to avoid light exposure to the incubation vials.

2.3. ThT Fluorescence Assay. ThT fluorescence assay was performed with a Hitachi F-7000 fluorescence spectrophotometer. An excitation wavelength of $450 \mathrm{~nm}$ with a slit width of $5 \mathrm{~nm}$ and an emission wavelength of $486 \mathrm{~nm}$ with a slit width of $10 \mathrm{~nm}$ were used. The voltage of photomultiplier tube was set to $700 \mathrm{~V}$. The concentration of ThT solution is $10 \mu \mathrm{M}$. The buffer is $20 \mathrm{mM}$ phosphate buffer at $\mathrm{pH}=7.4$. The assay was performed ex situ. To measure fibrillation kinetics, aliquots of HEWL incubation solutions without or with rifampicin were taken out of the incubation vial at different time-points and were subjected to the assay immediately. For each measurement, $40 \mu \mathrm{L}$ of incubation solution was added into $960 \mu \mathrm{L}$ of ThT solution in a $1.0 \mathrm{~cm}$ quartz cuvette. The solution in the cuvette was shaken first before running

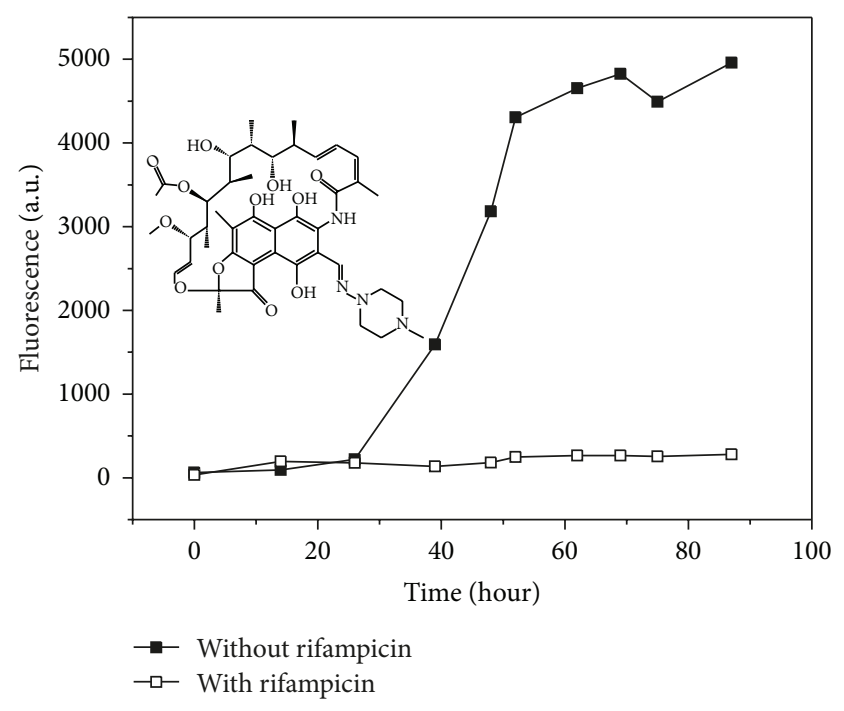

FIgURE 1: Effect of rifampicin on the fibrillation of HEWL by ThT fluorescence assay. Inset: molecular structure of rifampicin.

each acquisition. For rifampicin interference experiment, $40 \mu \mathrm{L}$ of incubation solution was added into $960 \mu \mathrm{L}$ of ThT solution in a $1.0 \mathrm{~cm}$ quartz cuvette first to obtain an initial ThT fluorescence reading at $486 \mathrm{~nm}$. Then $1.5 \mu \mathrm{L}$ of $7 \mathrm{mM}$ rifampicin solution was injected into the above solution and the change of ThT fluorescence reading at $486 \mathrm{~nm}$ over time was recorded.

2.4. AFM Measurement. All the AFM images were taken on dried samples in air with NT-MDT Solver P47 Scanning Probe Microscope (Zelenograd, Russia) in tapping mode. A $90 \mu \mathrm{m} \times 90 \mu \mathrm{m}$ scanner was used throughout the AFM experiment. The silicon cantilevers were purchased from NTMDT. The cantilever has a resonance frequency of $100 \mathrm{kHz}$ and a nominal force constant of $3 \mathrm{~N} / \mathrm{m}$. The samples for AFM measurement were prepared using the following method: $10 \mu \mathrm{L}$ incubation solution was diluted 100 times with deionized water (Millipore, Billerica, USA) and then $100 \mu \mathrm{L}$ of the diluted solution was dropped onto freshly cleaved mica. After 10 min waiting time, the protein solution was rinsed off with deionized water. The mica surface was then left dry under ambient condition.

2.5. FTIR Measurement. The FTIR spectra were obtained at room temperature with a Bruker Vertex 70 FTIR spectrometer (Bruker, Germany) equipped with a DLaTGS detector. The sample compartment was constantly purged with dry air from a home-made purge system to minimize water vapor interference. A demountable liquid cell with a pair of $\mathrm{CaF}_{2}$ windows and a $50 \mu \mathrm{m}$ spacer was utilized. Spectra were collected with a spectral resolution of $4 \mathrm{~cm}^{-1}$ and 128 scans. FTIR data processing was performed with OPUS software (Bruker, Germany). Prior hydrogen-deuterium (H/D) exchange on the HEWL was not performed before incubation in order to minimize structural perturbation on the protein. When HEWL in $\mathrm{D}_{2} \mathrm{O}$ solution was incubated at $62^{\circ} \mathrm{C}$, 


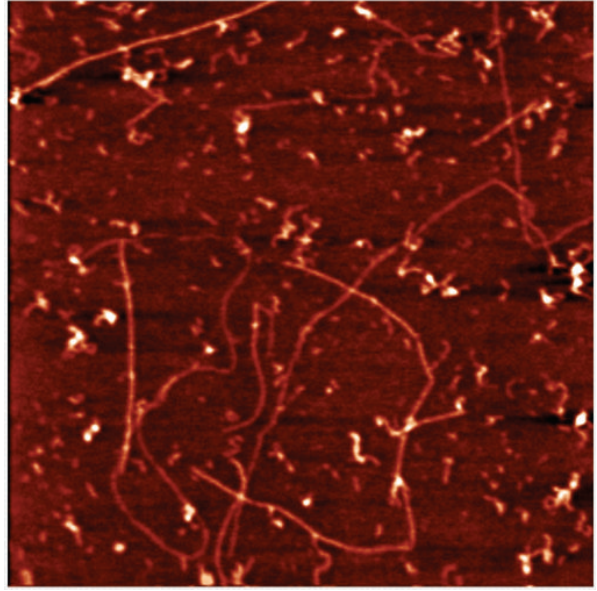

(a)

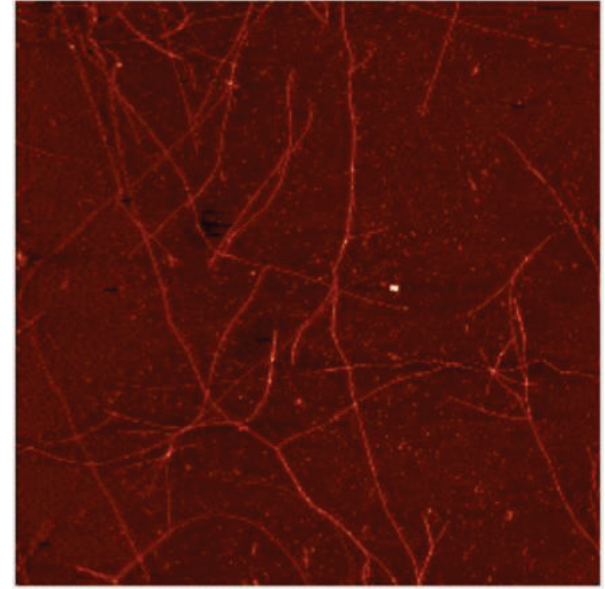

(b)

FIGURE 2: AFM characterization of HEWL fibrillation (a) without rifampicin in the incubation solution and (b) with rifampicin in the incubation solution. Scan size: $5 \mu \mathrm{m} \times 5 \mu \mathrm{m}$.

the peptide backbone became fully deuterated during the lag phase as evidenced by the disappearance of the amide I band at $1540 \mathrm{~cm}^{-1}$ after $5 \mathrm{~h}$ incubation [12]. Residual $\mathrm{H}_{2} \mathrm{O}$ in the overwhelming $\mathrm{D}_{2} \mathrm{O}$ forms $\mathrm{HOD}$ and has negligible effect on the spectral analysis in the amide I region.

\section{Results and Discussion}

We first use ThT fluorescence assay to investigate the inhibitory effect of rifampicin on the amyloid formation of HEWL. The ThT assay is the standard method to quantify the growth of fibrillar aggregates during amyloid formation $[13,14]$. ThT, as an extrinsic fluorescent probe molecule, once binding to amyloid fibrils, gives a characteristic fluorescent emission at $\sim 480 \mathrm{~nm}$ with $\sim 440 \mathrm{~nm}$ excitation. The intensity of ThT fluorescence is hypothesized to be proportional to the amount of amyloid fibrils. We here perform a comparative study on HEWL fibrillation without and with the presence of rifampicin in the incubation solution at $\mathrm{pD}=2$ and $62^{\circ} \mathrm{C}$ with the ThT assay. The heat and acidic condition are the standard conditions to induce fibril formation of HEWL [15]. At selected time-points, aliquots of incubation solution were taken out of the incubation vial for fluorescence measurements. The ThT assay results of this comparative study are shown in Figure 1. The fibrillation kinetic curve of HEWL without the influence of rifampicin shows a typical sigmoidal shape, featuring a lag phase, a growth phase, and an equilibrium phase. However, the fibrillation kinetic curve of HEWL under the influence of rifampicin is basically at the baseline level. A simple comparison between the two kinetic curves clearly suggests that rifampicin completely inhibited the fibrillation of HEWL under the current experimental conditions.

As already mentioned above, Meng et al. have shown that rifampicin could interfere with ThT assay. In fact, Hudson et al. also pointed out that ThT assay could be biased by exogenous compounds [16]. Therefore, to confirm the inhibitory

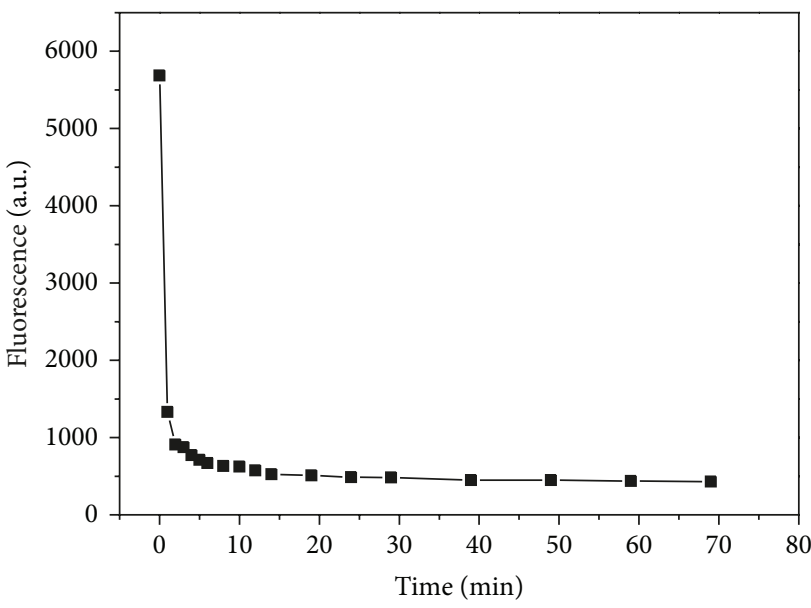

FIGURE 3: Fluorescence intensity decay of ThT upon the addition of rifampicin.

effect of rifampicin observed in the above ThT fluorescence investigation, we characterized the HEWL incubation solutions without and with rifampicin using AFM to see whether there are fibrillar aggregates present or not. Figure 2 gives the AFM results for HEWL incubation solutions with and without rifampicin. Surprisingly, on both of the samples, extensive presence of fibrillar aggregates was observed under AFM. This means that the AFM results do not support the conclusion that we drew based on ThT assay.

To explore the cause of the above conflicting evidences from ThT assay and AFM study, we performed the following experiments with fluorescence spectroscopy. We first added ThT into the solution containing HEWL fibrils and obtained the initial ThT fluorescence reading. Then, we added rifampicin into the above mixture and began to monitor ThT fluorescence decay over time. As shown in Figure 3, upon addition of rifampicin, there is an instant drop in ThT 


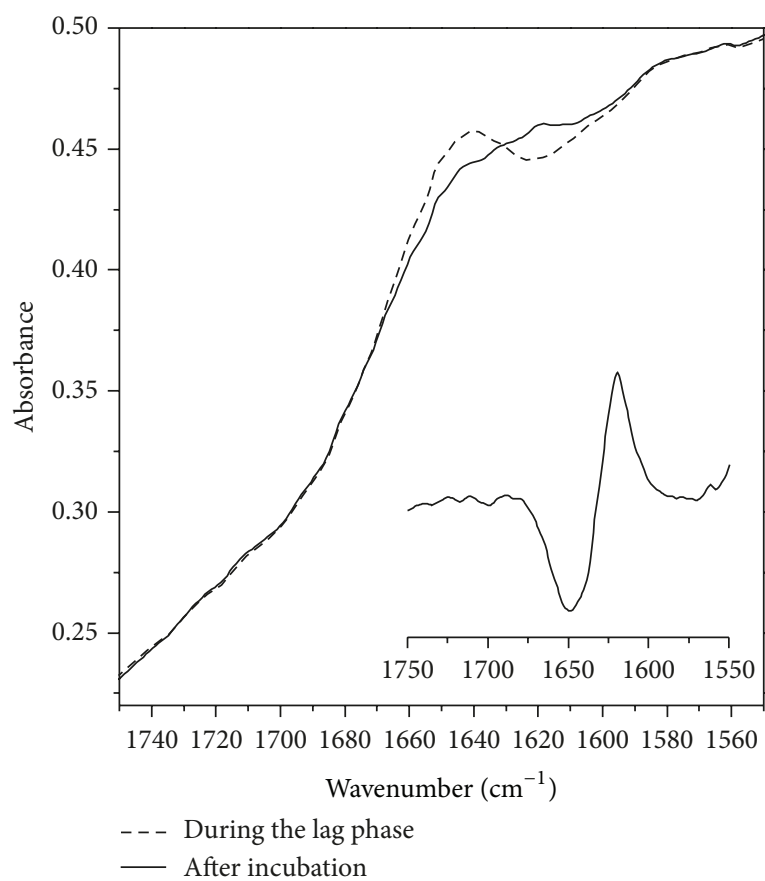

(a)

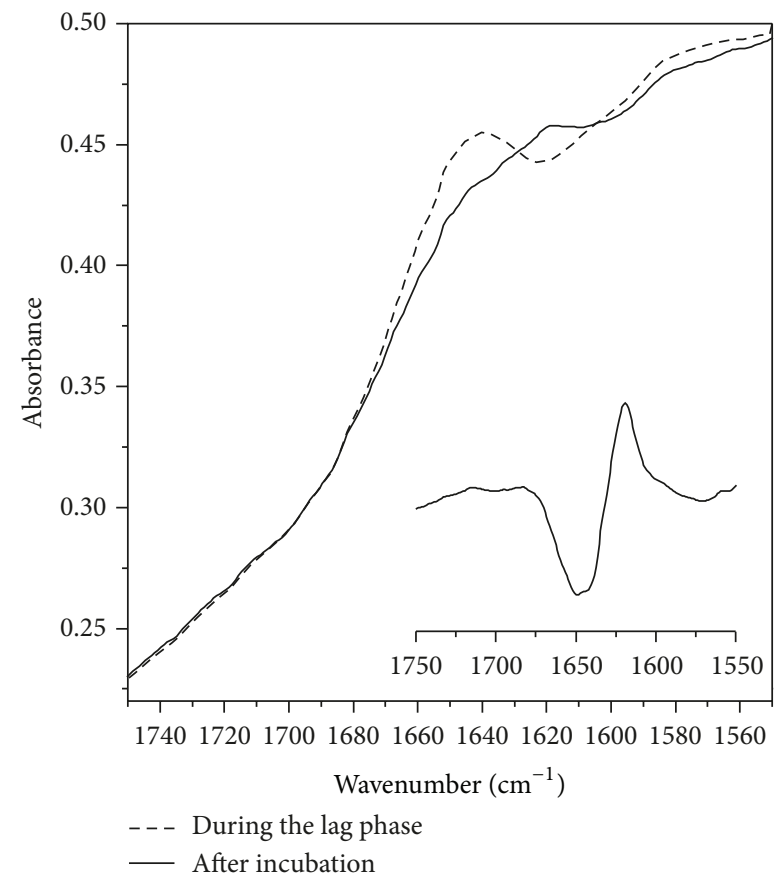

(b)

FIGURE 4: FTIR results for the inhibitory effect of rifampicin on HEWL fibrillation (a) without rifampicin in the incubation solution and (b) with rifampicin in the incubation solution. Dash line: raw spectrum taken during the lag phase; solid line: raw spectrum taken at the end of incubation. Inset: difference spectrum.

fluorescence intensity. Obviously, the presence of rifampicin interferes with ThT assay. The exact cause of this ThT intensity drop surely deserves future investigations, but it should be due to either quenching effect or rifampicin competition against ThT on available binding sites. Because of the interference of rifampicin, ThT assay cannot be used as a reliable method to evaluate the actual effect of rifampicin on HEWL fibrillation.

As ThT assay could give misleading false positive results, it is beneficial to search for alternative convenient methods for inhibitor screening. We would like to explore the feasibility of FTIR difference spectroscopy as an alternative inhibitor screening method. FTIR is a conformation sensitive technique due to the fact that each protein secondary structure has its unique infrared absorption in the $1700-1600 \mathrm{~cm}^{-1}$ amide I region [17]. This means that the growth of cross- $\beta$ structure during amyloid formation can be easily monitored and quantified with FTIR difference spectroscopy. In addition, as FTIR is a label free method and directly detects the $\beta$-sheet structures, it is basically immune to the interference of exogenous compounds such as rifampicin.

We performed two sets of FTIR measurements under identical conditions to explore the exact effect of rifampicin on the amyloid formation of HEWL. Figure 4(a) gives the raw spectra of HEWL incubation solution without the rifampicin presence taken during the lag phase and at the end of incubation. The difference spectrum between the two raw spectra is also shown in the inset. It is obtained by subtracting the FTIR spectrum of the incubation solution taken during the lag phase (i.e., at $5 \mathrm{~h}$ time-point) from that taken at the end of incubation (i.e., at $90 \mathrm{~h}$ time-point). The existence of the $1618 \mathrm{~cm}^{-1}$ peak in the difference spectrum is a direct indicator for the presence of amyloid fibril [12]. The intensity of this peak is directly proportional to the amount of HEWL fibril. Figure 4(b) gives the raw spectra of HEWL incubation solution with the rifampicin presence taken during the lag phase and at the end of incubation. The difference spectrum between the two raw spectra is also shown in the inset. The existence of the $1618 \mathrm{~cm}^{-1}$ peak shows that there is amyloid fibril presence even under the influence of rifampicin. This observation is consistent with the observation in the AFM study but contrary to the false positive result from ThT assay. Furthermore, we found that the intensity of the $1618 \mathrm{~cm}^{-1}$ fibril peak in the difference spectrum in Figure 4(b) is only slightly lower than that of the control shown in Figure 4(a). This supports the argument that rifampicin only marginally inhibits the amyloid formation of HEWL.

Clearly, Figure 4 gives a proof-of-concept example to support the proposal that FTIR difference spectroscopy can be used as an alternative inhibitor screening method. Certainly the FTIR assay used here still needs further optimizations in future studies. For example, the current transmission mode FTIR spectroscopy can be replaced with attenuate total reflection (ATR) mode FTIR spectroscopy. This can make 
sample handling and spectral subtraction more user-friendly. In addition, a high throughput FTIR accessory needs to be developed to make large-scale screening feasible.

\section{Conclusions}

In conclusion, we evaluated the inhibitory effect of rifampicin on the amyloid formation of hen egg white lysozyme (HEWL) with both ThT fluorescence assay and FTIR difference spectroscopy. Our results indicate that ThT assay is not a reliable assessment method as rifampicin interferes with such assay. Unlike ThT assay, FTIR difference spectroscopy directly monitors the growth of $\beta$-sheet structures during amyloid formation; thus, can be an accurate approach to evaluate the effect of rifampicin. With FTIR difference spectroscopy, we showed that rifampicin only had marginally inhibitory effect. We then proposed that FTIR difference spectroscopy can potentially be used as a convenient method for inhibitor screening in amyloid study.

\section{Conflict of Interests}

The authors declare that there is no conflict of interests regarding the publication of this paper.

\section{Acknowledgments}

The authors gratefully acknowledge the financial support from the National Natural Science Foundation of China (no. 21075027), the Natural Science Foundation of Hebei Province (no. B2011201082), the Key Project of Chinese Ministry of Education (no. 211014), and the Specialized Research Fund for the Doctoral Program of Higher Education (20121301110003).

\section{References}

[1] M. R. Sawaya, S. Sambashivan, R. Nelson et al., "Atomic structures of amyloid cross- $\beta$ spines reveal varied steric zippers," Nature, vol. 447, no. 7143, pp. 453-457, 2007.

[2] F. Chiti and C. M. Dobson, "Protein misfolding, functional amyloid, and human disease," Annual Review of Biochemistry, vol. 75, pp. 333-366, 2006.

[3] P. T. Lansbury and H. A. Lashuel, "A century-old debate on protein aggregation and neurodegeneration enters the clinic," Nature, vol. 443, no. 7113, pp. 774-779, 2006.

[4] J. D. Harper and P. T. Lansbury Jr., "Models of amyloid seeding in Alzheimer's disease and scrapie: mechanistic truths and physiological consequences of the time-dependent solubility of amyloid proteins," Annual Review of Biochemistry, vol. 66, pp. 385-407, 1997.

[5] C. G. Glabe, "Structural classification of toxic amyloid oligomers," The Journal of Biological Chemistry, vol. 283, no. 44, pp. 29639-29643, 2008.

[6] T. Tomiyama, S. Asano, Y. Suwa et al., "Rifampicin prevents the aggregation and neurotoxicity of amyloid $\beta$ protein in vitro," Biochemical and Biophysical Research Communications, vol. 204, no. 1, pp. 76-83, 1994.

[7] J. Li, M. Zhu, S. Rajamani, V. N. Uversky, and A. L. Fink, "Rifampicin inhibits $\alpha$-synuclein fibrillation and disaggregates fibrils," Chemistry and Biology, vol. 11, no. 11, pp. 1513-1521, 2004.
[8] J. J. Meier, R. Kayed, C.-Y. Lin et al., "Inhibition of human IAPP fibril formation does not prevent $\beta$-cell death: evidence for distinct actions of oligomers and fibrils of human IAPP," American Journal of Physiology: Endocrinology and Metabolism, vol. 291, no. 6, pp. E1317-E1324, 2006.

[9] V. H. Lieu, J. W. Wu, S. S.-S. Wang, and C.-H. Wu, "Inhibition of amyloid fibrillization of hen egg-white lysozymes by rifampicin and p-benzoquinone," Biotechnology Progress, vol. 23, no. 3, pp. 698-706, 2007.

[10] F. Meng, P. Marek, K. J. Potter, C. B. Verchere, and D. P. Raleigh, "Rifampicin does not prevent amyloid fibril formation by human islet amyloid polypeptide but does inhibit fibril thioflavin-T interactions: implications for mechanistic studies of $\beta$-cell death," Biochemistry, vol. 47, no. 22, pp. 6016-6024, 2008.

[11] K. P. Kumar and D. Chatterji, "Resonance energy transfer study on the proximity relationship between GTP binding site and the rifampicin binding site of Escherichia coli RNA polymerase," Biochemistry, vol. 29, no. 2, pp. 317-322, 1990.

[12] Y. Zou, Y. Li, W. Hao, X. Hu, and G. Ma, "Parallel $\beta$-sheet fibril and antiparallel $\beta$-sheet oligomer: new insights into amyloid formation of hen egg white lysozyme under heat and acidic condition from ftir spectroscopy," The Journal of Physical Chemistry B, vol. 117, pp. 4003-4013, 2013.

[13] H. LeVine III, "Quantification of $\beta$-sheet amyloid fibril structures with thioflavin T," Methods in Enzymology, vol. 309, pp. 274-284, 1999.

[14] L. P. Jameson, N. W. Smith, and S. V. Dzyuba, "Dye-binding assays for evaluation of the effects of small molecule inhibitors on amyloid (a $\beta$ ) self-assembly," ACS Chemical Neuroscience, vol. 3, pp. 807-819, 2012.

[15] R. Mishra, K. Sörgjerd, S. Nyström, A. Nordigården, Y.-C. Yu, and P. Hammarström, "Lysozyme amyloidogenesis is accelerated by specific nicking and fragmentation but decelerated by intact protein binding and conversion," Journal of Molecular Biology, vol. 366, no. 3, pp. 1029-1044, 2007.

[16] S. A. Hudson, H. Ecroyd, T. W. Kee, and J. A. Carver, "The thioflavin $\mathrm{T}$ fluorescence assay for amyloid fibril detection can be biased by the presence of exogenous compounds," FEBS Journal, vol. 276, no. 20, pp. 5960-5972, 2009.

[17] A. Barth and C. Zscherp, "What vibrations tell us about proteins," Quarterly Reviews of Biophysics, vol. 35, no. 4, pp. 369430, 2002. 

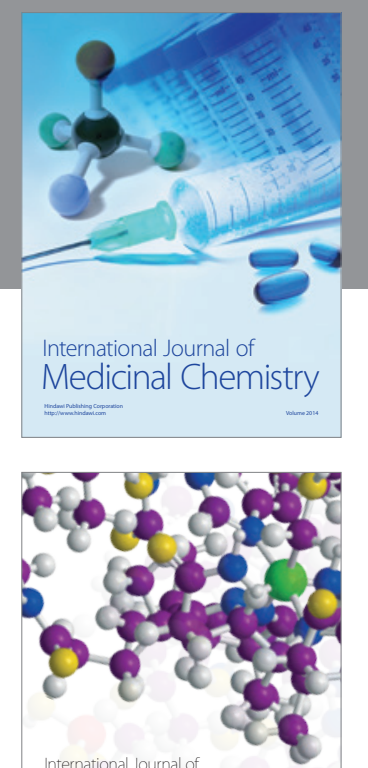

\section{Carbohydrate} Chemistry

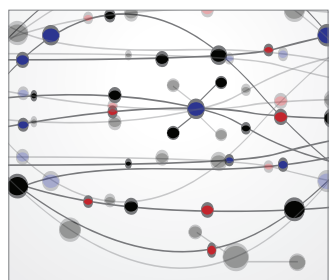

The Scientific World Journal
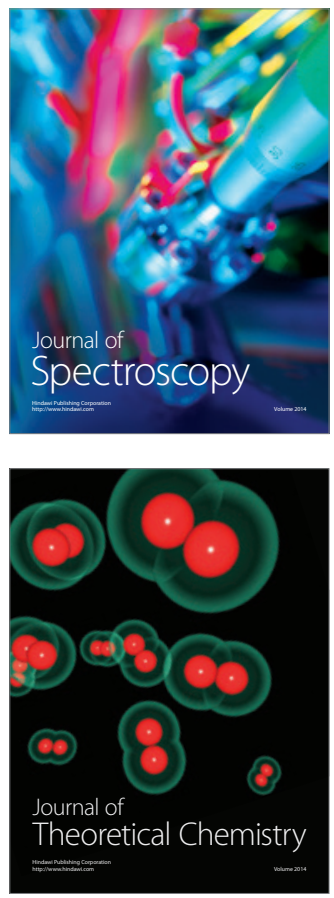
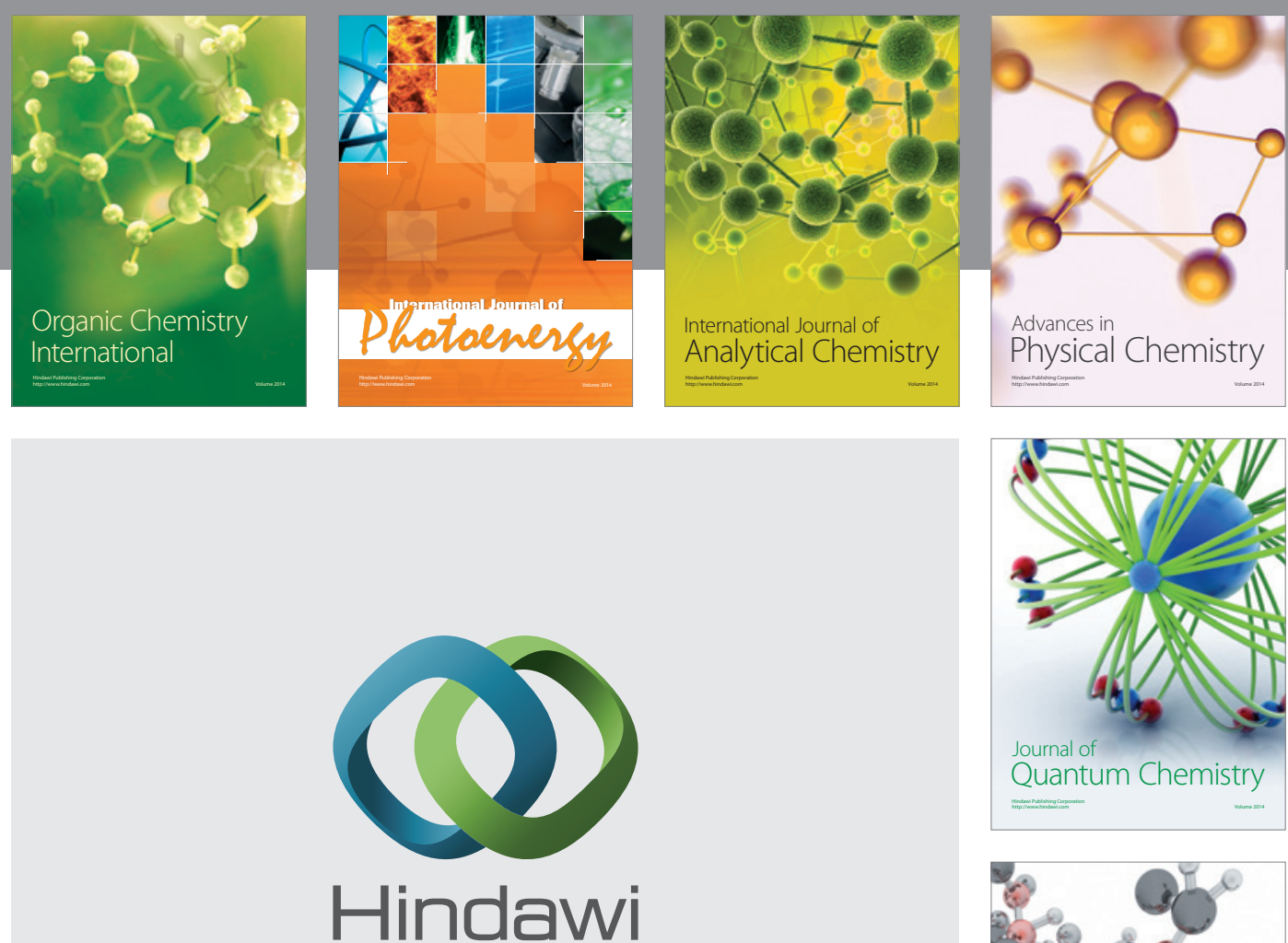

Submit your manuscripts at

http://www.hindawi.com

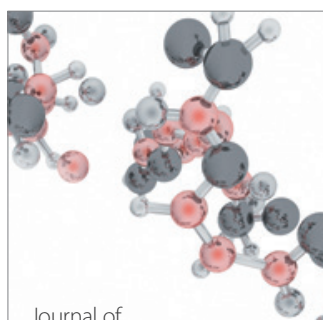

Analytical Methods

in Chemistry

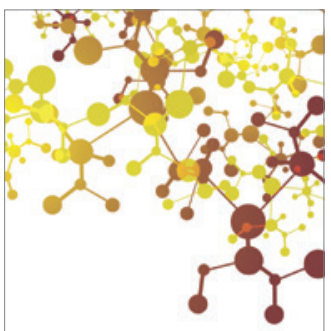

Journal of

Applied Chemistry

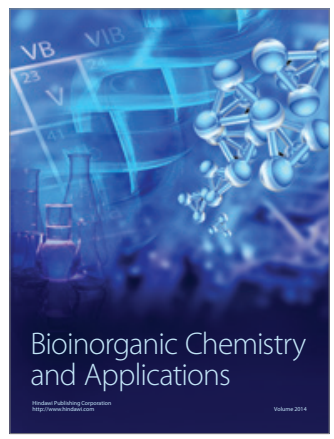

Inorganic Chemistry
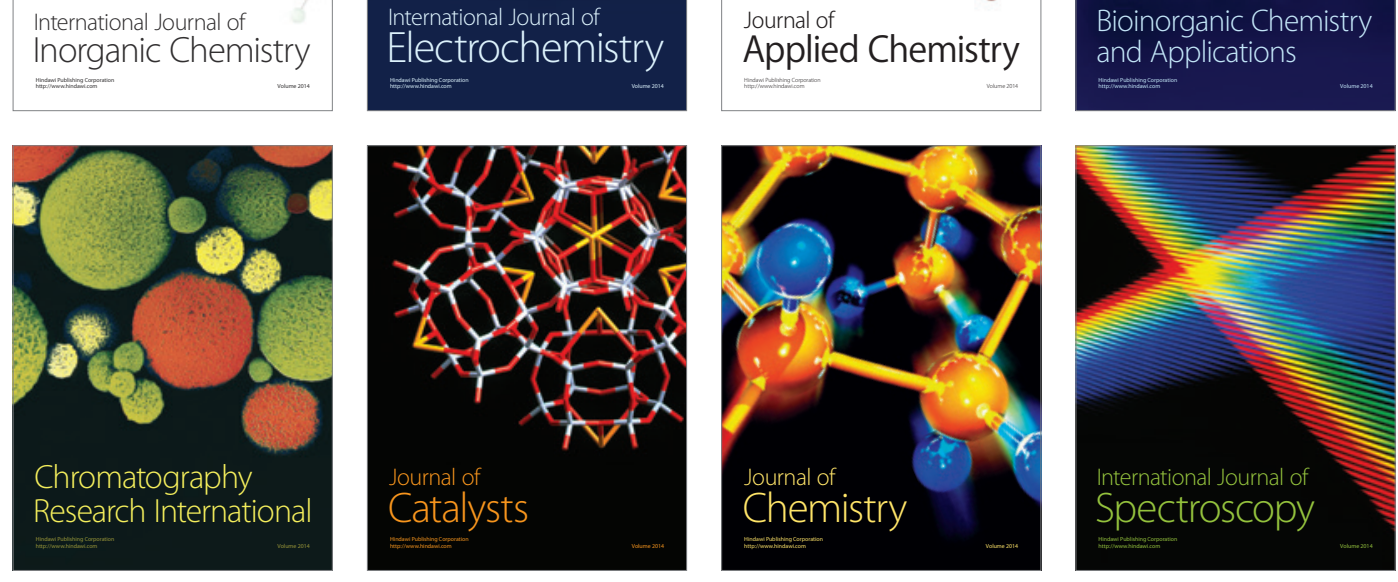